\title{
Design of optimised linear quadratic regulator for capsule endoscopes based on artificial bee colony tuning algorithm
}

\author{
Ibrahim Khalaf Mohammed, Abdulla Ibrahim Abdulla \\ Department of Systems and Control Engineering, University of Ninevah, Mosul, REPUBLIC OF IRAQ \\ e-mails: ibrahimallehaby16@gmail.com;abd_63mo@yahoo.com
}

\section{SUMMARY}

Wireless Capsule Endoscope (WCE) is a new medical device that can be used for examining the whole digestive tract if effectively actuated. In this paper, a new three-coil actuator is proposed for the capsule endoscope navigation system. The proposed system, which is based on the currentcontrolled magnetic levitation concept, utilises a small permanent magnet within the capsule body and an arrangement of controlled electromagnet actuator placed on a movable frame. The dynamics of the proposed control system is modelled mathematically and then formulated in state space form. In this research, the Linear Quadratic Regulator (LQR) technique is used for designing a 3DOF controller for the capsule actuation system. Artificial Bee Colony (ABC) tuning algorithm is used for obtaining optimum values for controller gain parameters. The optimised $L Q R$ controller is simulated by using the Matlab/Simulink tool, and its performance is then evaluated based on the stability and control effort parameters to validate the proposed system. Finally, the simulation results suggest that the LQR controller based on the ABC optimisation method can be adopted to synthesise an effective capsule actuation system.

KEY WORDS: $\quad$ wireless capsule endoscope (WCE); electromagnetic actuator; linear quadratic regulator ( $L Q R)$; artificial bee colony $(A B C)$.

\section{INTRODUCTION}

The WCE is a new diagnostic device provided by a digital camera and is used for examining the whole gastrointestinal tract without discomfort or need for sedation that traditional procedures cannot [1]. However, its passive movement mode limits its use as a commercial diagnosis tool in hospitals. Therefore, there is an incredible interest from endoscopists and bio-engineers in the methods of controlled actuation for these medical devices. Magnetic locomotion is highly recommended by many researchers in order to move the capsule in the digestive tract wirelessly due to the fact that a patient's body, which has very small magnetic permeability, does not have an effect on the distribution of the magnetic field. Furthermore, 
the magnet does not need to connect to a power supply to move [2]. Simi et al. [3] proposed a new miniaturised hybrid endoscopic capsule for the digestive tract evaluation. They fabricated a prototype capsule-type micro robot with an active hybrid locomotion system which merges with an internal actuation mechanism with external magnetic dragging. The internal actuator is based on a micro motor, whereas the external actuator is based on the magnetic interaction between a set of small cylindrical permanent magnets inserted in the capsule and a big external cylindrical permanent magnet mounted on a passive hydraulic arm. The practical tests based on the in vitro tests showed that the proposed hybrid capsule could proceed with a maximum moving speed of $8 \mathrm{~cm} / \mathrm{min}$. However, the actuation of the actuator was not validated for capsule functions and the supply capacity of the inserted battery. Additionally, further miniaturisation for the capsule size is required in order to achieve an ingestible capsule size.

In 2012, a new actuation approach was proposed by Yim et al. [4, 5] for a capsule endoscope. They fabricated a prototype of a compliant magnetic robot-driven capsule based on medical procedures in the stomach. The device is actuated and oriented magnetically based on a rolling locomotion method. They implanted two permanent magnets in both ends of a dummy capsule and used the force and torque of an external permanent magnet connected across a motor to rotate the capsule. However, the actuator force was not controlled to manipulate the capsule in the free space of the tested organ.

Lien et al. [6] proposed a magnetic actuation system for enabling the surgeons to control the mobility of the endoscopic capsule by moving their own hands as previously proposed by [7]. They fabricated a new prototype capsule endoscope equipped with shorter and longer focal length lenses for the visualisation of close-up and far-end stomach wall. An external magnetic field navigator (MFN) based on a permanent magnetic rotor with a built-in stepping motor was fabricated to manoeuvre the capsule inside the stomach. However, the proposed device was not wireless, as it still needed to be connected to power and image data transfer cables.

A new handheld magnetic actuator for a spiral-type WCE is proposed by Ye et al. [8]. They fabricated an actuator mainly consisting of a permanent magnet attached to a step motor, a microcontroller and a dummy capsule with a cylindrical magnetic shell and a spiral. The spiraltype device can move across the rotational magnetic field produced by the handheld actuator. The ex-vivo experiments in porcine small bowel showed that the medical device can move forward or backward under the control of the actuator. However, the handheld actuator cannot orient the capsule in the desired direction. The capsule rotational movement could cause blurring in the captured images. The regulation signal applied to the step motor is not calculated based on the capsule position feedback. Hasson et al. [9] presented a prototype of a steering mechanism for the Tethered Capsule Endoscope (TCE) system used for examining the stomach. The navigation mechanism consists of a controller, an extension tube made of the flexible Bowden cables and a steering system. Three thin steel wires in $120^{\circ}$ configuration are placed in the tube and used for steering the capsule. In the steering unit, a mock-up capsule is connected to the adaptor cap of the Bowden cable. The experimental tests have shown that the TCE can be steered in the desired direction. However, the capsule cannot be used for examining all digestive organs. In addition, the navigation of the cabled device through the digestive tract can cause pain and discomfort to the patient.

In 2015, another electromagnetic actuation system for capsule endoscopy was introduced by Lee et al. [10]. The proposed system was used for achieving 3D locomotion and steering of the capsule endoscope in the digestive tract. The system utilised five pairs of coils and a permanent magnet inserted in the capsule endoscope. The external magnetic field exerted by 
the solenoid components on the embedded magnet is used for orienting and navigating the capsule within the digestive organs. In order to validate the actuation approach, the ex vivo tests are executed by using a small intestine extracted from a cow. However, the moving speed of the capsule by propulsion force of the actuator is not high enough. In addition, the capsule motion in the $z$-direction by using the system is helical, hence, it is possible that some important spots in the tested organ may not be scanned. Therefore, a new low cost wireless navigation system using magnetic actuator based on three coils is proposed in this paper to address the problems associated with the actuation systems introduced in the literature survey (capsule size, orientation, power supply, image blurring, feedback, cabling, speed etc). The actuator employs three coils fixed in $\left(120^{\circ}\right)$ and configured on a 3D movable frame for the purpose of applying magnetic force to the inserted magnet in the capsule in order to guide it through the desired 3D trajectory within the stomach and the bowel.

This study focuses on the design of a linear control system for moving the dipole in 3D within the area around the operating point and maintaining it at a desired position. The stabilised device can then navigate the intestine by manipulating the actuator frame and using three degrees of freedom (3DOF) robot. The introduced scheme is based on magnetic levitation, which seems appropriate for high precision motions if a robust tracking algorithm is applied to deal with its inherent instability problem [11]. The system is analysed mathematically to describe its dynamics, and then modelled in state space form to enable the use of the state feedback technique for the purpose of designing a controller. The controller is based on a linear quadratic regulator (LQR) technique, which is highly recommended for precision movement applications. In order to validate the controller, the actuation system is simulated by using the Matlab/Simulink tool, and its performance is then evaluated based on overshoot, settling time and control input parameters.

However, the use of this control method includes many drawbacks, such as the limitation of the state and control variable by applying the trial-and-error procedure in order to determine the control gain coefficients. Therefore, there is a considerable interest in optimisation algorithms used to obtain optimum parameters values for state feedback controller techniques. For LQR controller design, there are many computer-aided optimisation methods used for finding optimum weighting matrices elements such as genetic algorithm (GA) [12], particle swarm optimisation (PSO) [13], particle swarm inspired evolutionary algorithm (PSEA) [14], and artificial bee colony (ABC) [15].

$\mathrm{ABC}$ method is one of the tuning algorithms that has been successfully applied to solve various types of optimisation problems [16]. Tuning performance of the $A B C$ algorithm is compared to that of GA and PSO method for the roll control system problem based on LQR technique of an aircraft [17]. Basturk and Karaboga compared the performance of ABC optimisation algorithm with those of PSO and PS-EA [15], GA [18] and DE, PSO and EA [19] on various test problems. In this research, the $\mathrm{ABC}$ tuning algorithm was developed for the capsule actuation system by using the LQR technique. The performance of the ABC-LQR controller is analysed with respect to the step input based on standard control criteria, which includes maximum overshoot, rise time (time necessary to reach $90 \%$ of its final value), settling time (time necessary to reach $95 \%$ of its final value), steady state error and control input.

The paper is organised as follows. Section II presents system configuration. In section III, the modelling and the dynamics of the proposed system are formulated. Tracking system technique and tuning method are described in section IV. Section V introduces strategy of the 
capsule actuation system. In section VI, simulation results of the navigation system are presented and followed by conclusions and prospective research in section VII.

\section{SYSTEM CONFIGURATION}

The preliminary setup of the proposed 3DOF actuation system is depicted in Figure 1. The scheme mainly consists of a controlled electromagnet, which is assembled by using three ironcored coils (radius $r_{c}$, length $L_{c}$ ) fixed symmetrically on a 3DOF Cartesian coordinate slide, a small cylindrical permanent magnet (radius $r_{d}$, length $L_{d}$ ) which is made of an alloy of neodymium, iron, and boron $\mathrm{NdFeB}$ enclosed by a capsule with a generating coil (wound around the capsule body), three-position coil sensors attached to lower poles of the actuator coils, a 3D simulated bowl tube, a processor to implement the controller of the proposed actuation system, and a 3DOF robot to move the coil frame.

In the proposed capsule navigation system, the embedded magnet served as the actuation source, whereas the capsule coil based on AC magnetic field was used as a localisation source. The purpose of using the $\mathrm{AC}$ magnetic field is to discriminate the capsule localisation field with the DC actuation field of the coil. The magnetic sensors are used to provide the controller with feedback information about the capsule position, which is used for adjusting the actuator current required to move the capsule in the $x, y$ and $z$-direction and maintain it at the desired position.

\section{SYSTEM MODELLING AND DYNAMICS}

The force vectors of the system in the free space are illustrated in Figure 2(a). The orientation definition of the coil and magnet in the Spherical coordinate system is shown in Figures 2(b) and (c) respectively. The figure shows the inclination angles $\beta$ and $\alpha$ and azimuth angles $\theta$ and $\gamma$ of the coil and magnet respectively.

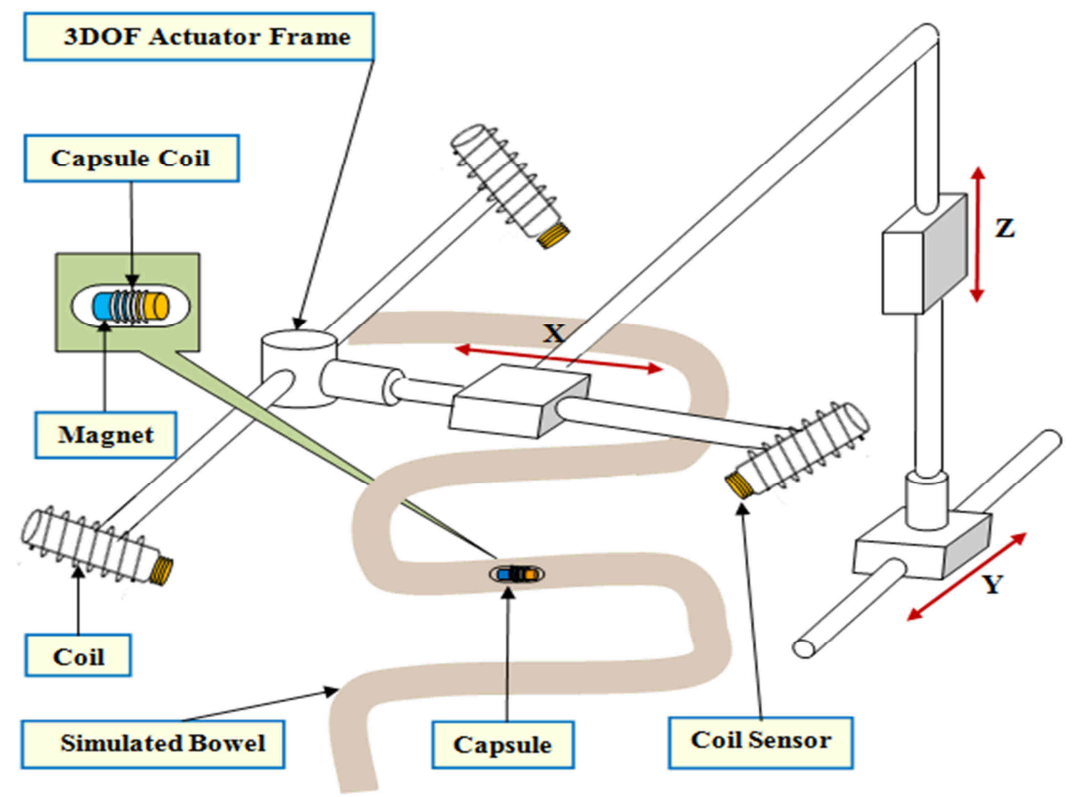

Fig. 1 Conceptual platform of the proposed control system 


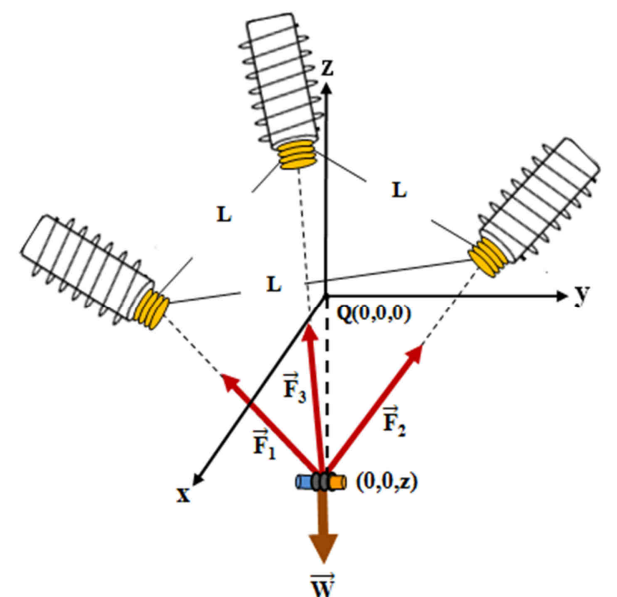

(a)

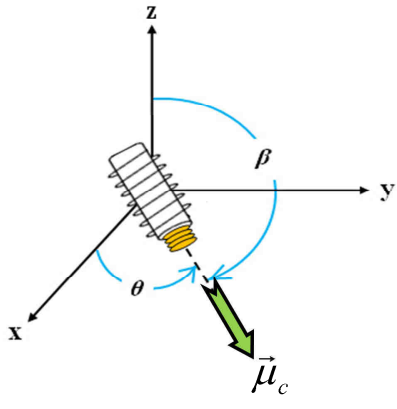

(b)

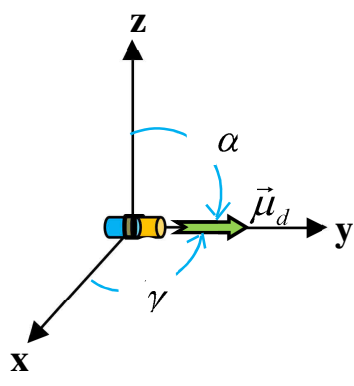

(c)

Fig. 2 Geometry of the system in the free space

(a) Force vectors of the system; (b) Orientation angles of the coil; (c) Orientation angles of the magnet

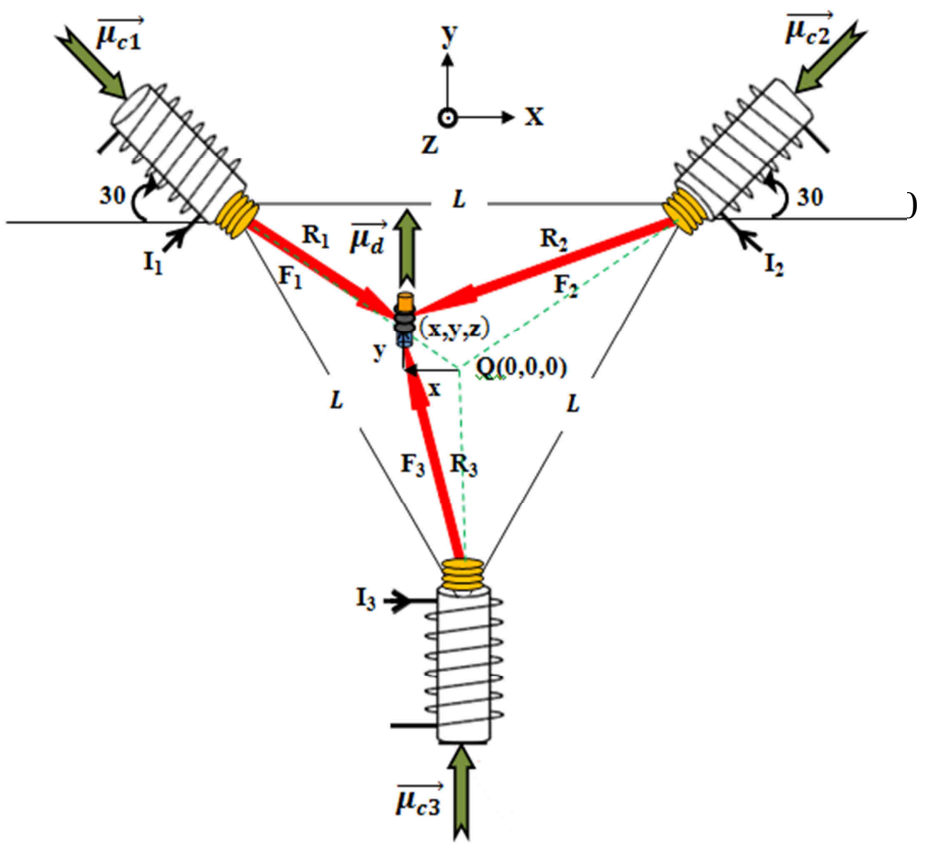

Fig. 3 Schematic diagram of the proposed control system in the xy-plane

In the proposed scheme, the actuator is set on the 3D movable frame so that the inclination angles of the coil 1,2 and 3 have the same value of $135^{\circ}$, while their azimuth angles are $210^{\circ}$, $330^{\circ}$ and $90^{\circ}$, respectively. Figure 3 illustrates a 2D schematic diagram of the actuation system. In this study, the inclination $\alpha$ and azimuth $\gamma$ angles of the dipole are assumed to have the same value of $90^{\circ}$. The diagram shows the projections of the distance and the force vectors of the control system in the $x y$-plane. 
The magnetic force, produced by a coil, and exerted on the embedded dipole, is provided in expression Eq. (1).

$$
\vec{F}=\frac{3 \mu_{0} \mu_{r}}{4 \pi}\left\{\frac{\left(\vec{\mu}_{c} \cdot \vec{R}\right) \vec{\mu}_{d}+\left(\vec{\mu}_{d} \cdot \vec{R}\right) \vec{\mu}_{c}+\left(\vec{\mu}_{c} \cdot \vec{\mu}_{d}\right) \vec{R}}{R^{5}}-\frac{5\left(\vec{\mu}_{c} \cdot \vec{R}\right)\left(\vec{\mu}_{d} \cdot \vec{R}\right) \vec{R}}{R^{7}}\right\}
$$

The definition of the above equation parameters are as follows: $\mu_{0}$ is air magnetic permeability (Tm/A), $\mu_{r}$ is the relative magnetic permeability of the coil core, $\vec{\mu}_{c}$ is magnetic moment vector of a coil $\left(A m^{2}\right), \vec{\mu}_{d}$ is a magnetic moment vector of the magnet $\left(A m^{2}\right), \vec{R}$ is the vector distance from coil to the dipole $(\mathrm{m})$ and $R$ is the distance between coil and the magnet (m).

Based on Figure 2(b) and (c), the magnetic moment of the coil $i(i=1,2,3)$ and dipole is provided by:

$$
\begin{gathered}
\mu_{c i}=N_{c i} I_{i} A_{c i} \\
\mu_{d}=\frac{B V}{\mu_{o}} \\
\vec{\mu}_{c i}=\mu_{c i}\left(\sin \left(\beta_{i}\right) \cos \left(\theta_{i}\right) \hat{x}+\sin \left(\beta_{i}\right) \sin \left(\theta_{i}\right) \hat{y}+\cos \left(\beta_{i}\right) \hat{z}\right) \\
\vec{\mu}_{d}=\mu_{d}(\sin (\alpha) \cos (\gamma) \hat{x}+\sin (\alpha) \sin (\gamma) \hat{y}+\cos (\alpha) \hat{z})
\end{gathered}
$$

where $\mu_{c i}$ and $\mu_{d}$ is the magnetic moment value of the coil $i$ and magnet respectively, $N_{c i}, I_{i}$ and $A_{c i}$ is the number of turns, current $(A)$ and the cross-section area of the coil $i\left(\mathrm{~m}^{2}\right)$, respectively, $B$ is the intrinsic induction of the magnet $(T)$, and $V$ is the volume of the magnet $\left(\mathrm{m}^{3}\right), \hat{x}, \hat{y}, \hat{z}$ is the unit standard vectors in the $x, y$ and $z$-direction respectively. If the coils have the same magnetic and physical parameters, then the magnet moment value of the coils is the same $\mu_{c i}=N_{c} I A_{c}$. Based on the mentioned above values of inclination and azimuth angle of coils and the magnet and using Eqs. (2c) and (2d), the magnetic moment vectors of the system are provided by:

$$
\begin{gathered}
\vec{\mu}_{c 1}=\frac{N_{c} I A_{c}}{\sqrt{2}}\left(-\frac{\sqrt{3}}{2} \hat{x}-\frac{1}{2} \hat{y}-\hat{z}\right) \\
\vec{\mu}_{c 2}=\frac{N_{c} I A_{c}}{\sqrt{2}}\left(\frac{\sqrt{3}}{2} \hat{x}-\frac{1}{2} \hat{y}-\hat{z}\right) \\
\vec{\mu}_{c 3}=\frac{N_{c} I A_{c}}{\sqrt{2}}(0 \hat{x}+\hat{y}-\hat{z}) \\
\vec{\mu}_{d}=\mu_{d}(0 \hat{x}+\hat{y}+0 \hat{z})
\end{gathered}
$$

The response of air core coil sensor is easy to characterise by Faraday's fundamental law of induction, as follows:

$$
V_{s}=-\mu_{0} N_{s} \frac{d \varphi}{d t}
$$

where $V_{s}$ is the induced voltage $(V), N_{s}$ is the number of coil turns, and $\varphi$ is the magnetic flux passing through a coil. The above expression refers to the fact that induced voltage in the $N_{s}$ turn coil winding is equal to the rate of change of electromagnetic flux. Generally, the 
sensitivity of an air coil sensor is relatively low, hence in order to improve the output response, a ferromagnetic core with high permeability should be used. Based on Eq. (4), the output voltage of an iron-cored coil sensor is governed as follows:

$$
V_{s}=-\mu_{0} \mu_{r c} N_{s} A \frac{d H}{d t}
$$

where $A$ is the cross-sectional area of the coil sensor $\left(\mathrm{m}^{2}\right), \mu_{r c}$ is the resultant permeability of the core and $H$ is the magnetic field intensity $(A / m)[20]$ :

$$
\mu_{r c}=\frac{\mu_{r s}}{1+D_{f}\left(\mu_{r s}-1\right)}
$$

where $\mu_{r s}$ is the permeability of the iron core of the coil sensor, and $D_{f}$ is the demagnetisation factor. It is worth considering that the measurement of the capsule position in this study is based on the air core coil sensor. The reliability of the controller design depends on the robustness of the transfer function, and to achieve this, the modelling of the system must be based on realistic parameter values. In this research, the realistic parameters values of the system, which are listed in Table 1, are taken from previous work [21].

By using Eq. (1), the movement of the magnet due to magnetic force produced by the system actuator (three electromagnetic coils) in the $x, y$ and $z$-direction is governed by Newton's second law, and it can be expressed as follows:

$$
\begin{gathered}
\frac{d^{2}(x, y, z)}{d t^{2}}=\frac{3 \mu_{0} \mu_{r}}{4 \pi M} \sum_{i=1}^{3}\left\{\frac{\left(\vec{\mu}_{c i} \cdot \vec{R}_{i}\right) \vec{\mu}_{d}+\left(\vec{\mu}_{d} \cdot \vec{R}_{i}\right) \vec{\mu}_{c i}+\left(\vec{\mu}_{c i} \cdot \vec{\mu}_{d}\right) \vec{R}_{i}}{R_{i}^{5}}-\right. \\
\left.-\frac{5\left(\vec{\mu}_{c i} \cdot \vec{R}_{i}\right)\left(\vec{\mu}_{d} \cdot \vec{R}_{i}\right) \vec{R}_{i}}{R_{i}^{7}}\right\}-\frac{\vec{W}}{M} \\
\frac{d^{2} x}{d t^{2}}=\left.\frac{d^{2}(x, y, z)}{d t^{2}}\right|_{\hat{x} \text {-direction }} \\
\frac{d^{2} y}{d t^{2}}=\left.\frac{d^{2}(x, y, z)}{d t^{2}}\right|_{\hat{y} \text {-direction }} \\
\frac{d^{2} z}{d t^{2}}=\left.\frac{d^{2}(x, y, z)}{d t^{2}}\right|_{\hat{z}-\text { direction }}
\end{gathered}
$$

where:

$\vec{\mu}_{c 1}=\left(\mu_{c 1} / \sqrt{2}\right)(-\sqrt{3} \hat{x} / 2-\hat{y} / 2-\hat{z}), \vec{\mu}_{c 2}=\left(\mu_{c 2} / \sqrt{2}\right)(\sqrt{3} \hat{x} / 2-\hat{y} / 2-\hat{z})$,

$\vec{\mu}_{c 3}=\left(\mu_{c 3} / \sqrt{2}\right)(0 \hat{x}+\hat{y}-\hat{z})$ are the magnetic moment vectors of coil1, coil2 and coil3, respectively,

$\vec{\mu}_{d}=\mu_{d}(0 \hat{x}+\hat{y}+\hat{z}), \vec{W}=M g(O \hat{x}+O \hat{y}-\hat{z})$ is the weight vector of the inserted dipole,

$M$ is the mass of the embedded magnet $(\mathrm{kg})$,

$g$ is the gravitational acceleration $\left(\mathrm{m} / \mathrm{s}^{2}\right)$,

$\vec{R}_{1}=(L / 2+x) \hat{x}+(-L /(2 \sqrt{3})+y) \hat{y}+z \hat{z}, \vec{R}_{2}=(-L / 2+x) \hat{x}+(-L /(2 \sqrt{3})+y) \hat{y}+z \hat{z}$, 
$\vec{R}_{3}=x \hat{x}+(L / \sqrt{3}+y) \hat{y}+z \hat{z}$ is the distance between the inserted magnet and coil1, coil2 and coil3 respectively.

Assuming that the electromagnets have the same magnetic and physical parameters, i.e. $K_{1}=K_{2}=K_{3}=K$, the $x$-direction movement formula of the inserted magnet based on Eqs. (7a) and $(7 \mathrm{~b})$ can be written as:

$$
\begin{aligned}
\ddot{x}(t)= & \frac{K}{M}\left\{I_{1}\left[\frac{\left(\sqrt{3} y_{1}-x_{2}\right)}{R_{1}^{5}}-\frac{5 x_{2} y_{1} D_{1}}{R_{1}^{7}}\right]-I_{2}\left[\frac{\left(\sqrt{3} y_{1}+x_{1}\right)}{R_{2}^{5}}-\frac{5 x_{1} y_{1} D_{1}}{R_{2}^{5}}\right]+\right. \\
& \left.+2 I_{3}\left[\frac{x}{R_{3}^{5}}-\frac{5 x y_{2} D_{3}}{R_{3}^{5}}\right]\right\}
\end{aligned}
$$

Table 1 Physical and magnetic parameters of the control system

\begin{tabular}{cccc}
\hline Parameter & Value & Parameter & Value \\
\hline$L$ & $0.06 \mathrm{~m}$ & $M$ & $0.009 \mathrm{~kg}$ \\
$N$ & 250 & $B$ & $1 \mathrm{~T}$ \\
$r_{c}$ & $0.0125 \mathrm{~m}$ & $\mu_{r}$ & 200 \\
$r_{d}$ & $0.006 \mathrm{~m}$ & $\mu_{0}$ & $4 \pi \cdot 10^{-7}(\mathrm{Tm} / \mathrm{A})$ \\
$L_{r}$ & $0.012 \mathrm{~m}$ & $K$ & $1.987 \cdot 10^{-6}\left(\mathrm{Nm}^{4} / \mathrm{A}\right)$ \\
\hline
\end{tabular}

where $K=3 \mu_{r} B V N A_{c} / 16 \pi$ is a force constant depending on the geometry of the system $\left(\mathrm{Nm}^{4} / \mathrm{A}\right)$. The definition of the variables $\left(x_{1}, x_{2}, y_{1}, y_{2}, D_{1}, D_{2}, D_{3}\right)$ is included in Appendix A.1.

It is worth noting that the above force formula is non-linear, and that it involves three position variables $(x, y, z)$ and three current variables $\left(I_{1}, I_{2}, I_{3}\right)$. In this study, the proposed control system is linear. Therefore, for scheme design purpose, Eq. (8) is linearized by using Taylor's series expansion about suitable equilibrium values $\left(x_{0}, y_{0}, z_{0}, I_{01}, I_{02}, I_{03}\right)$. It is worth considering that the desired equilibrium position is at the centre of the actuator frame $\left(x_{0}=0, y_{0}=0\right)$ and vertical distance $\left(z_{0}\right)$ as much as possible while keeping the equilibrium input $\left(I_{01}, I_{02}, I_{03}\right)$ within the acceptable values.

Let us consider the perturbation about these equilibrium values as follows: $\Delta x(t)=x(t)-x_{0}$, $\Delta y(t)=y(t)-y_{0}, \quad \Delta z(t)=z(t)-z_{0}, \quad \Delta I_{1}(t)=I_{1}(t)-I_{01}, \quad \Delta I_{2}(t)=I_{2}(t)-I_{02}$, $\Delta I_{3}(t)=I_{3}(t)-I_{03}$ where $\Delta x(t), \Delta y(t), \Delta z(t), \Delta I_{1}(t), \Delta I_{2}(t), \Delta I_{3}(t)$ are the position and current deviation from the equilibrium states $\left(x_{0}, y_{0}, z_{0}, I_{01}, I_{02}, I_{03}\right)$, respectively.

Based on the above assumption, after algebraic manipulation, the linearized model of the dipole movement about the operating values $\left(x_{0}, y_{0}, z_{0}, I_{01}, I_{02}, I_{03}\right)$ in the $x$-direction can be described by:

$$
\Delta \ddot{x}(t)=\sum_{i=1}^{3} C_{1 i} \Delta I_{i}(t)+C_{14} \Delta x(t)+C_{15} \Delta y(t)+C_{16} \Delta z(t)
$$

The expressions for the above equation parameters $C_{1 i}(i=1,2, \ldots 6)$ are included in Appendix B.1. 
In the $y$-direction, the movement of the inserted dipole can be expressed on the basis of Eqs. (7a) and (7c) as follows:

$$
\begin{aligned}
\ddot{y}(t)= & \frac{K}{M}\left\{I_{1}\left[\frac{\sqrt{3}\left(x_{2}-\sqrt{3} y_{1}-2 z\right)}{R_{1}^{5}}-\frac{5 y_{1}^{2} D_{1}}{R_{1}^{7}}\right]-I_{2}\left[\frac{\sqrt{3}\left(x_{1}-\sqrt{3} y_{1}-2 z\right)}{R_{2}{ }^{5}}-\frac{5 y_{1}^{2} D_{1}}{R_{2}{ }^{7}}\right]+\right. \\
& \left.+2 I_{3}\left[\frac{\left(3 y_{2}-\sqrt{3} z\right)}{R_{3}{ }^{5}}-\frac{5 y_{2}{ }^{2} D_{3}}{R_{3}{ }^{5}}\right]\right\}
\end{aligned}
$$

For linear controller implementation purposes, the above dynamic equation is linearized around the equilibrium values $\left(x_{0}, y_{0}, z_{0}, I_{01}, I_{02}, I_{03}\right)$ as follows:

$$
\Delta \ddot{y}(t)=\sum_{i=1}^{3} C_{2 i} \Delta I_{i}(t)+C_{24} \Delta x(t)+C_{25} \Delta y(t)+C_{26} \Delta z(t)
$$

where the expressions for the elements $C_{2 i}(i=1,2, \ldots 6)$ are outlined in Appendix B.2.

In the $z$-direction, the movement of the embedded magnet is expressed on the basis of Eqs. (7a) and (7d) as follows:

$$
\begin{aligned}
\ddot{z}(t)= & \frac{K}{M}\left\{-I_{1}\left[\frac{\left(2 \sqrt{3} y_{1}+z\right)}{R_{1}^{5}}-\frac{5 y_{1} z_{1} D_{1}}{R_{1}^{7}}\right]-I_{2}\left[\frac{\left(2 \sqrt{3} y_{1}+z\right)}{R_{2}^{5}}-\frac{5 y_{1} z_{1} D_{2}}{R_{2}{ }^{7}}\right]+\right. \\
& \left.+2 I_{3}\left[\frac{\left(-\sqrt{3} y_{2}+z\right)}{R_{3}^{5}}-\frac{5 y_{2} z D_{3}}{R_{3}{ }^{5}}\right]\right\}-g
\end{aligned}
$$

Linearization of Eq. (12) about the operating point $\left(x_{0}, y_{0}, z_{0}, I_{01}, I_{02}, I_{03}\right)$ can be described by:

$$
\Delta \ddot{z}(t)=\sum_{i=1}^{3} C_{3 i} \Delta I_{i}(t)+C_{34} \Delta x(t)+C_{35} \Delta y(t)+C_{36} \Delta z(t)
$$

where the expressions for $C_{3 i}(i=1,2, \ldots 6)$ are outlined in Appendix B.3. From the free body diagram in Figure 2(a), at the equilibrium state, the resultant magnetic force exerted by the electromagnetic coil on the levitated dipole in the $x$ and $y$-direction is zero, whereas in the $z$ direction it equals the capsule weight $W$. Based on Eqs. (8) and (10), the current inputs $\left(I_{01}, I_{02}, I_{03}\right)$ of the system at the equilibrium point $\left[x_{0}, y_{0}, z_{0}\right]^{T}=[0,0,0]^{T}$ are the same. In order to satisfy the equilibrium condition, the currents that pass in the actuator coils must be the same. Based on Eq. (12), the excitation currents of the scheme can be calculated by using the following equation:

$$
I_{0 i}=\frac{M g}{1.3854 \cdot 10^{-6} K}, \quad i=1,2,3
$$

It this study, the input of the system at equilibrium case is reduced as much as possible as the inserted magnet is placed in the plane of the actuator coils $\left(x_{0}=0, y_{0}=0, z_{0}=0\right)$.

\section{STATE SPACE FORMULATION}

In order to design a 3DOF actuator, the modelling of the proposed system should be formulated in state space form $\Delta \boldsymbol{X}(n x 1)=\left[x_{1}, x_{2}, x_{3}, x_{4}, x_{5}, x_{6}\right]^{T}=[\Delta x, \Delta \dot{x}, \Delta y, \Delta \dot{y}, \Delta z, \Delta \dot{z}]^{T}$ being the state vector of the system, the applied coil currents are considered the control 
input's vector so that $\Delta \boldsymbol{U}(m \times 1)=\left[\Delta I_{1}, \Delta I_{2}, \Delta I_{3}\right]^{T}$, and the movement of the magnet in the $x y z$ planes is considered the output's vector so that $\Delta \boldsymbol{Y}(p x 1)=\left[y_{1}, y_{2}, y_{3}\right]^{T}=[\Delta x, \Delta y, \Delta z]^{T}$. The state and the output equation of the proposed Linear Time-Invariant (LTI) system can be written based on Eqs. (9), (11) and (13) as in Eqs. (15) and (16), respectively.

$$
\begin{aligned}
& \Delta \dot{\boldsymbol{X}}(t)=\boldsymbol{A} \Delta \boldsymbol{X}(t)+\boldsymbol{B} \Delta \boldsymbol{U}(t) \\
& \Delta \boldsymbol{Y}(t)=\boldsymbol{C} \Delta \boldsymbol{X}(t)+\boldsymbol{D} \Delta \boldsymbol{U}(t)
\end{aligned}
$$

where $\boldsymbol{A}$ is the system matrix $(n \times n), \boldsymbol{B}$ is the input matrix $(n \times m), \boldsymbol{C}$ is the output matrix $(p \times n)$, and $\boldsymbol{D}$ is feed forward matrix $(p \times m)$. These matrices are provided by:

$$
\begin{aligned}
& \boldsymbol{A}=\left[\begin{array}{cccccc}
0 & 1 & 0 & 0 & 0 & 0 \\
C_{14} & 0 & C_{15} & 0 & C_{16} & 0 \\
0 & 0 & 0 & 1 & 0 & 0 \\
C_{24} & 0 & C_{25} & 0 & C_{26} & 0 \\
0 & 0 & 0 & 0 & 0 & 1 \\
C_{34} & 0 & C_{35} & 0 & C_{36} & 0
\end{array}\right], \quad \boldsymbol{B}=\left[\begin{array}{ccc}
0 & 0 & 0 \\
C_{11} & C_{12} & C_{13} \\
0 & 0 & 0 \\
C_{21} & C_{22} & C_{23} \\
0 & 0 & 0 \\
C_{31} & C_{32} & C_{33}
\end{array}\right], \\
& \boldsymbol{C}=\left[\begin{array}{llllll}
1 & 0 & 0 & 0 & 0 & 0 \\
0 & 0 & 1 & 0 & 0 & 0 \\
0 & 0 & 0 & 0 & 1 & 0
\end{array}\right], \quad \boldsymbol{D}=\left[\begin{array}{lll}
0 & 0 & 0 \\
0 & 0 & 0 \\
0 & 0 & 0
\end{array}\right]
\end{aligned}
$$

\section{OPTIMISED CONTROLLER APPROACH}

In this section, the theory of the controller technique used for designing the proposed capsule navigation system and the $\mathrm{ABC}$ tuning algorithm method, which is adopted to obtain optimum values for the controller gain parameters, are presented.

\subsection{CONTROLLER DESIGN METHOD}

In this paper, a more common technique LQR is adopted to implement the proposed 3D controller, since it basically seeks a compromise between the minimum energy and the best performance. The LQR controller can be successfully implemented in this application as the proposed system is observable and state controllable. For any desired input, this approach involves applying the optimal control input vector:

$$
\Delta \boldsymbol{U}(t)=\boldsymbol{N} \boldsymbol{X}_{d}(t)-\boldsymbol{K}_{o} \Delta \boldsymbol{X}(t)
$$

where $\boldsymbol{N}$ is a feed-forward scaling matrix, $\boldsymbol{K}_{o}$ is the optimal feedback gain matrix, and $\boldsymbol{X}_{d}(t)$ is the desired state vector to track the input commands and keep the magnet in the desired position, while minimizing the quadratic cost function [22]:

$$
J=\int_{0}^{\infty}\left[\boldsymbol{e}^{T}(t) \boldsymbol{Q}(t) \boldsymbol{e}(t)+\Delta \boldsymbol{U}^{T}(t) \boldsymbol{R}(t) \Delta \boldsymbol{U}(t)\right] d t
$$

where $\boldsymbol{Q}(t)$ and $\boldsymbol{R}(t)$ are referred to as combined state and control penalty matrices, respectively. The parameters of the controller matrices are determined by trial and error so that the controller can achieve a compromise between the applied input and the state magnitude of the system $[23,24]$. In this study, so as to avoid the drawback of the trial and 
error procedure, an optimisation algorithm based on the ABC tuning method is adopted to find optimum controller gain parameters.

The feedback gain matrix $\boldsymbol{K}_{o}$ can be calculated by solving the following Riccati equation and relation Eqs. (19) and (20) by using the Matlab software:

$$
\begin{gathered}
\boldsymbol{A}^{T} \boldsymbol{P}+\boldsymbol{P A}-\boldsymbol{P} \boldsymbol{B} \boldsymbol{R}^{-1} \boldsymbol{B}^{T} \boldsymbol{P}+\boldsymbol{Q}=0 \\
\boldsymbol{K}_{0}=\boldsymbol{R}^{-1} \boldsymbol{B}^{T} \boldsymbol{P}
\end{gathered}
$$

The controller weighting matrices $\boldsymbol{Q}(t)$ and $\boldsymbol{R}(t)$ are set so that the control system can achieve the desired performance based on a reasonable control effort.

It is worth considering that based on the weighting matrices $\boldsymbol{Q}(t)$ and $\boldsymbol{R}(t)$, which govern the system states behavior and input currents respectively, the feedback gain matrix $\boldsymbol{K}_{o}$ is calculated by using the Matlab command "lqr", whereas the pre-compensator $N$ is obtained on the basis of the state and output matrices $(\boldsymbol{A}, \boldsymbol{B}, \boldsymbol{C}, \boldsymbol{D})$ and controller gain $\boldsymbol{K}_{0}$ from the Matlab function, i.e. the designer's function in m.file code.

\subsection{CONTROLLER TUNING METHOD}

In this study, an optimisation algorithm $\mathrm{ABC}$ is adopted to implement the tuning process for the LQR weighting matrices $\boldsymbol{Q}$ and $\boldsymbol{R}$, which is used for calculating the feedback gain matrix $\boldsymbol{K}_{\boldsymbol{o}}$ of the actuation system. A set of good control parameters $\boldsymbol{Q}$ and $\boldsymbol{R}$ can achieve a good output response for the system, and result in the minimisation of performance criteria in the time domain including the rise time $t_{r}$, settling time $t_{s}$, maximum overshoot (\%Mo) and steady state error $e_{s s}$. In Bees Algorithm, the colony of artificial bees consists of three groups of bees: employed bees, onlookers and scouts.

The first half of the colony consists of employed artificial bees, and the second half includes the onlookers. For every food source, there is only one employed bee. In other words, the number of employed bees is equal to the number of food sources around the hive. The employed bee whose food source has been abandoned by the bees becomes a scout. The position of a food source represents a possible solution to the optimisation problem, and the nectar amount of a food source corresponds to the quality (fitness) of the associated solution. The number of the employed bees or the onlooker bees is equal to the number of solutions in the population [25].

The flow chart of the $\mathrm{ABC}$ tuning algorithm used for obtaining the optimum gain parameters for the proposed LQR controller system is shown in Figure (4), where SN is the number of food source, $D$ is the dimension of the problem for optimisation of the LQR controller system [namely $\boldsymbol{Q}$ diagonal elements: $q_{11}, q_{22}, q_{33}, q_{44}, q_{55}, q_{66}$ and $\boldsymbol{R}$ diagonal elements: $r_{11}, r_{22}, r_{33}$ ] and $\mathrm{MCN}$ is the maximum cycle number.

\section{STRATEGY OF THE ACTUATION SYSTEM}

The main idea of the proposed system is that the magnet, which is enclosed in the device as a marker for localisation, can also be moved by the magnetic force of the external controlled magnetic field produced by coils placed outside the human body on a movable frame. 


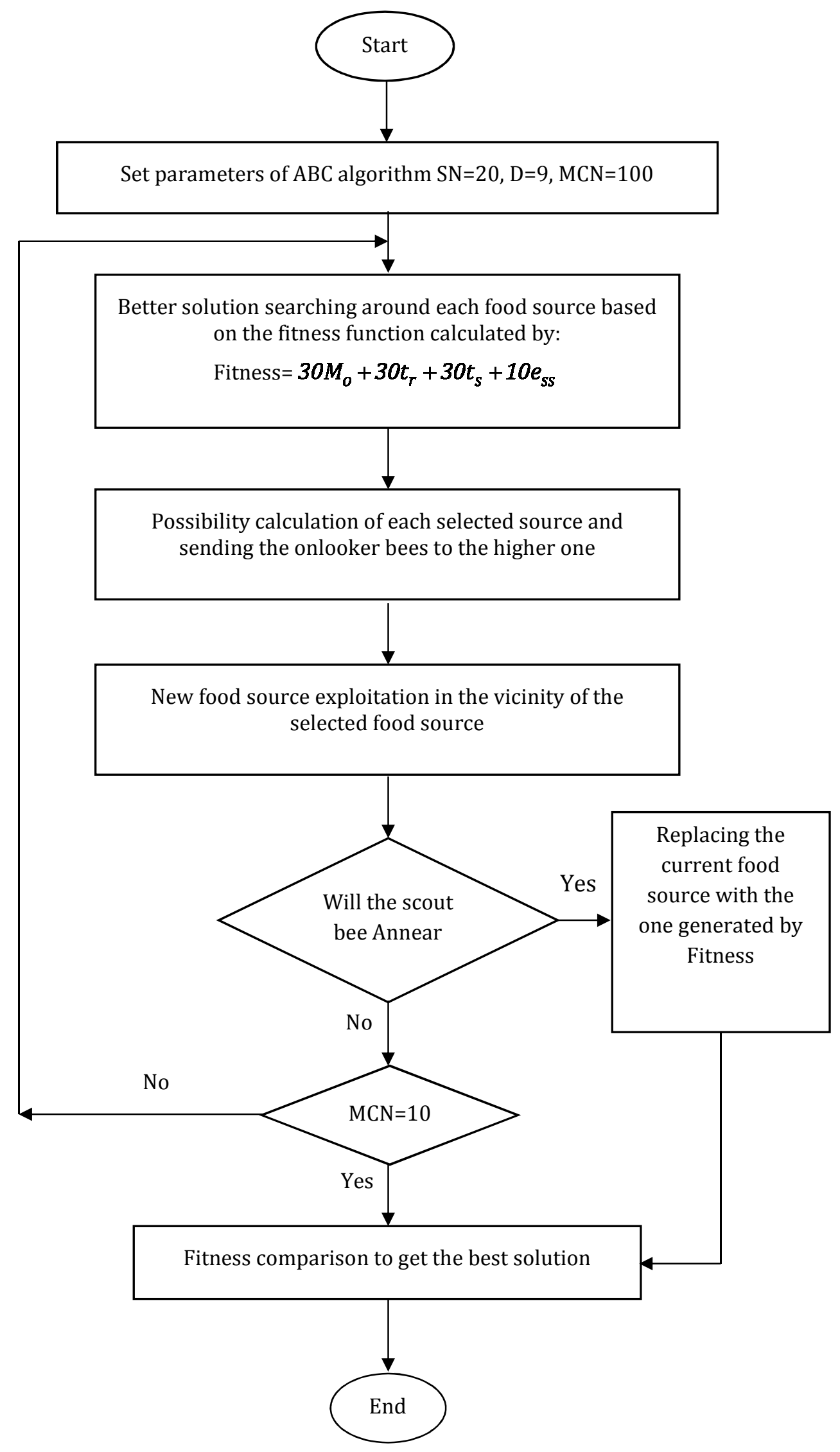

Fig. 4 Flow chart of the $A B C$ optimization algorithm 
The procedure of the proposed actuation system for the medical device in the digestive tract includes the application of the following steps:

- Step1: Applying an auto tuning LQR controller to move the inserted magnet across a distance $\Delta x(t), \Delta y(t), \Delta z(t)$ within the area around the operating point to set the dipole at the desired position relative to the movable electromagnet frame.

- Step 2: Navigate the embedded magnet through the simulated intestine in the $x, y$ and $z$ direction by moving the actuator frame and/or the patient's bed using the robotic frame manipulator.

This study focuses on the design of a linear 3DOF tracking system to achieve step 1. It is worth noting that it can successfully implement the 3DOF LQR controller for the proposed capsule actuation system as the system based on $\boldsymbol{A}, \boldsymbol{B}$ and $\boldsymbol{A}, \boldsymbol{C}$ matrices, and the use of both Matlab commands "ctrb" and "obsv" is completely controllable and completely observable. The block diagram of the control system is shown in Figure 5. The idea presented here for the proposed scheme is very simple, as it has been based on the magnetic levitation concept, which is highly recommended for accurate movement applications. The system is analysed mathematically and modelled in state space form to enable the use of the state feedback LQR technique to design the controller. In this research, the $A B C$ tuning algorithm is used to optimise the controller through obtaining the optimum value for its weighting matrices elements. It is worth noting that the scaling matrix $\boldsymbol{N}$ is added to the system as shown in Figure (5) to scale the desired input $\boldsymbol{X}_{d}(t)$, so that the system output will be equal to the reference input in steady state condition [2].

The feedback gain $\boldsymbol{K}_{o}$ of the controller is calculated on the basis of the optimised weighting matrices $\boldsymbol{Q}, \boldsymbol{R}$ and state $\boldsymbol{A}$ and input $\boldsymbol{B}$ matrices of the system based on the Matlab command "lqr". In the proposed positioning system, the coil sensors attached to the actuator coils are used for obtaining the device position information from the AC magnetic field generated by the capsule coil which is decoupled from the DC magnetic actuation field. Based on the feedback sensors information, the controller is designed to manipulate the current inputs for the purpose of guiding the dipole to the desired region and keep it there. The stabilised device then navigates the bowel by moving the frame and/or the patient.

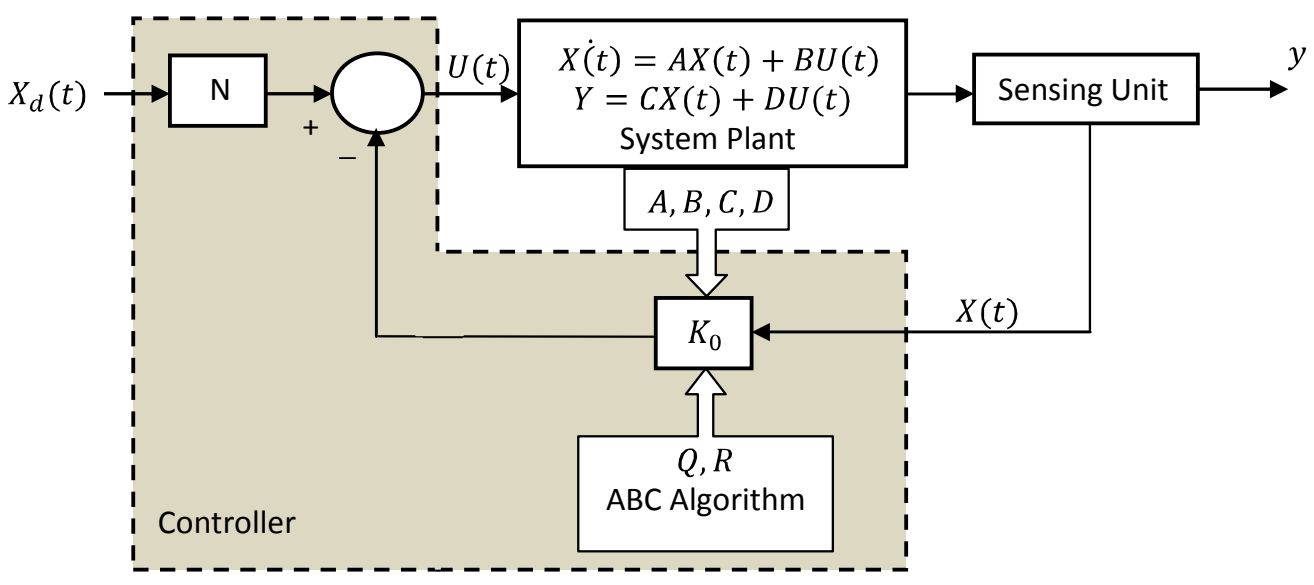

Fig. 5 Block diagram of the proposed control system 
Regarding the system control effort, the coil currents, which are governed by the initial position of the capsule and the physical and magnetic parameters of the system, must be within a reasonable value (no more than several amperes). The reason for this is that the increasing current heats the coil thus changing the magnetic behaviour of the actuator.

\section{SIMULATION AND RESULTS OF THE LQR CONTROLLER}

For the proposed LTI system as stated in Eqs. (15) and (16), based on the realistic values in Table 1, the currents and the state matrices $\boldsymbol{A}, \boldsymbol{B}$ as the elements of the system required to track the desired input are calculated and listed in Table 2.

In this section, the Matlab/Simulink environment is used as the simulation tool to verify the performance of the proposed control system based on rise time, settling time, maximum overshoot, and control input parameters. The LQR controller is designed for the desired response with rise time $t_{r}$ of $0.1 \mathrm{~s}$, settling time $t_{s}$ of $0.5 \mathrm{~s}$, and maximum overshoot $M o$ of $5 \%$. The tracking scheme is designed to guide the embedded magnet with the initial state vector $\boldsymbol{X}_{i}(t)=\left[x_{i}, \dot{x}_{i}, y_{i}, \dot{y}_{i}, z_{i}, \dot{z}_{i}\right]^{T}=[0,0,0,0,0,0]^{T}$ to a position represented by the desired states vector $\left[\Delta x_{d}, \Delta y_{d}, \Delta z_{d}\right]^{T}=[1,1,1]^{T}$.

Table 2 Electrical and linearisation parameters of the control system

\begin{tabular}{cccc}
\hline Parameter & Value & Parameter & Value \\
\hline$I_{0 i}, i=1,2,3$ & $32 \mathrm{~mA}$ & $C_{24}$ & 0 \\
$C_{11}$ & $201.5 \mathrm{~N} /(\mathrm{kg} \mathrm{A})$ & $C_{25}$ & $-2983.5 \mathrm{~N} /(\mathrm{kg} \mathrm{m})$ \\
$C_{12}$ & $368.46 \mathrm{~N} /(\mathrm{kg} \mathrm{A})$ & $C_{26}$ & $2086 \mathrm{~N} /(\mathrm{kg} \mathrm{m})$ \\
$C_{13}$ & 0 & $C_{31}$ & $-197.46 \mathrm{~N} /(\mathrm{kg} \mathrm{A})$ \\
$C_{14}$ & $-2777 \mathrm{~N} /(\mathrm{kg} \mathrm{m})$ & $C_{32}$ & $-197.46 \mathrm{~N} /(\mathrm{kg} \mathrm{A})$ \\
$C_{15}$ & 0 & $C_{33}$ & $89 \mathrm{~N} /(\mathrm{kg} \mathrm{A})$ \\
$C_{16}$ & 0 & $C_{34}$ & 0 \\
$C_{21}$ & $147 \mathrm{~N} /(\mathrm{kg} \mathrm{A})$ & $C_{35}$ & $2086 \mathrm{~N} /(\mathrm{kg} \mathrm{m})$ \\
$C_{22}$ & $147 \mathrm{~N} /(\mathrm{kg} \mathrm{A})$ & $C_{36}$ & $-2922.8 \mathrm{~N} /(\mathrm{kg} \mathrm{m})$ \\
$C_{23}$ & $177 \mathrm{~N} /(\mathrm{kg} \mathrm{A})$ & & \\
\hline
\end{tabular}

In practical application, the system operates through the placement of the inserted magnet initially in the vicinity of the operating point, as the proposed linear controller is valid only around the equilibrium position. It is worth considering that closing the distance between the initial and final position of the capsule keeps the current values of actuator within the acceptable range.

In this research, the scaling matrix $\boldsymbol{N}$ is included into the proposed system so that, based on the optimum controller gain values, it can guide the states of the system output through the desired trajectory and normalise them to the demand values $[1,1,1]^{T}$. Figure 6 presents the Simulink model of the proposed capsule actuation system. Tracking performance of the system 


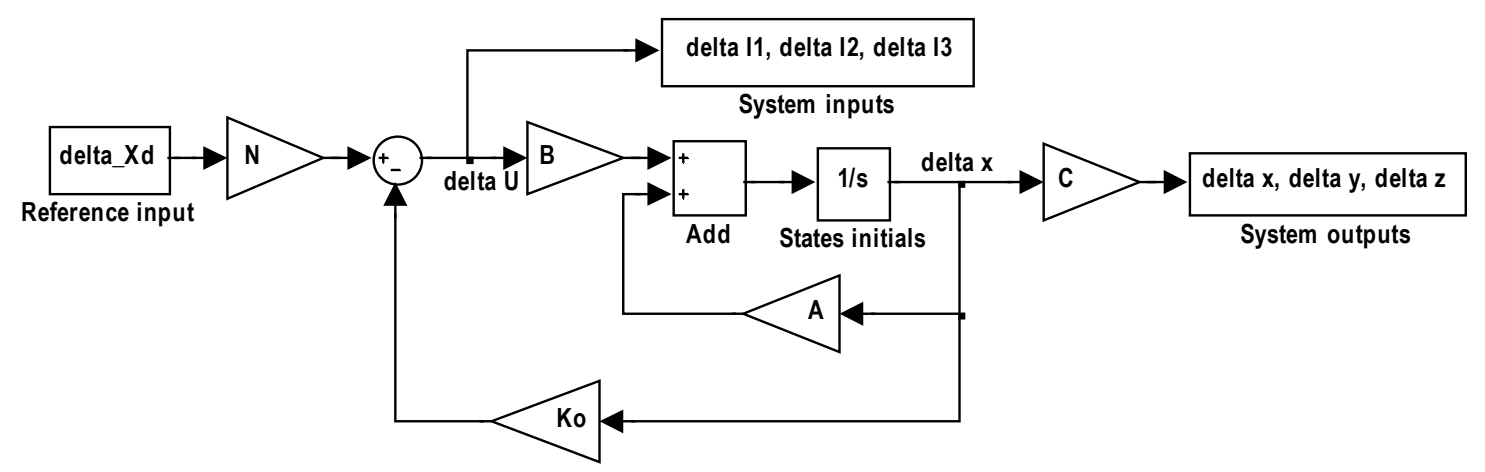

Fig. 6 Simulation model of the proposed LQR tracker system

can be examined by a proper setting of the controller gain matrix $\boldsymbol{K}_{o}$ and the scaling matrix $\boldsymbol{N}$ which determines the control input $\Delta \boldsymbol{U}(t)$ and eliminates the steady state error capability, respectively. The optimal weighting vectors $\boldsymbol{Q}$ and $\boldsymbol{R}$ obtained for the LQR controller by using the tuning algorithm $\mathrm{ABC}$ are provided by:

$\boldsymbol{Q}=\operatorname{blkdiag}\left(q_{11}, q_{22}, q_{33}, q_{44}, q_{55}, q_{66}\right)$ and $\boldsymbol{R}=\operatorname{blkdiag}\left(r_{11}, r_{22}, r_{33}\right)$ where $q_{11}=313.399, q_{22}=1 e-9, q_{33}=0.0884, q_{44}=1 e-9, q_{55}=598.6766, q_{66}=1 e-9, r_{11}=1 e-9$, $r_{22}=1 e-9$, and $r_{33}=1 e-9$. These controller matrices elements are set to effectively enable the system states to track the desired step input while keeping the control inputs as small as possible. Based on the controller weighting matrices $\boldsymbol{Q}, \boldsymbol{R}$, the feedback gain matrix $\boldsymbol{K}_{o}$ was calculated by using the Matlab command "lqr" as follows:

$$
\boldsymbol{K}_{o}=\left[\begin{array}{llllll}
103919.9 & -7.9 & 6068 & 1.9 & -572061.5 & -57.4 \\
520249.6 & 59.9 & -3375.4 & -1.1 & -63349.5 & 16.2 \\
178678.8 & 36.6 & 6323.2 & 2.0 & 517116.1 & 63.3
\end{array}\right]
$$

whereas the scaling matrix $\boldsymbol{N}$ was calculated on the basis of the system dynamic matrices $(\boldsymbol{A}, \boldsymbol{B}, \boldsymbol{C}, \boldsymbol{D})$ and the controller feedback gain matrix $\boldsymbol{K}_{o}$, which is tuned optimally by the $\mathrm{ABC}$ algorithm, by using the defined Matlab function in m.file code; $\boldsymbol{N}=\operatorname{rescale}\left(\boldsymbol{A}, \boldsymbol{B}, \boldsymbol{C}, \boldsymbol{D}, \boldsymbol{K}_{o}\right)$.

$$
\boldsymbol{N}=\left[\begin{array}{lll}
-462074.2 & 453524.1 & 702117.5
\end{array}\right]^{T}
$$

The time response of the system output $\Delta x(t), \Delta y(t), \Delta z(t)$ based on the above gain parameters is shown in Figures 7(a), (b) and (c), respectively. Figures 8(a), (b), and (c) represent the input currents $\Delta I_{1}(t), \Delta I_{2}(t)$ and $\Delta I_{3}(t)$ of the actuation system, respectively. It is observed that the optimised LQR controller based on the ABC tuning algorithm can effectively achieve a fast and stable response with a reasonable control effort. Based on the mini plots of Figures 7(a), (b) and (c), the system output $\Delta x(t), \Delta y(t)$ and $\Delta z(t)$ followed the desired unit step trajectories with no overshoot, approximately zero steady state error, rise time of approximately $80 \mathrm{~ms}$ and settling time of approximately $95 \mathrm{~ms}$.

Regarding the control effort, it can be seen from the mini figure of the input signals as shown in Figures 8 (a), (b), (c) that the initial and steady-state control input of the system were within acceptable values. Figures 9 and 10 present the converging elements of the LQR weighting matrices $\boldsymbol{Q}$ and $\boldsymbol{R}$ respectively based on the ABC optimisation method through iterations. 
It is worth considering that, in order to increase the maximum levitation distance of the capsule to a realistic value, the magnetic force of the actuator exerted on the inserted magnet should be increased. In order to achieve that, the control law can be supported by increasing the number of turns of the coil and by using high permeability iron-cored coils. However, increasing the number of turns can cause a rise in the coil conductivity which will increase the settling time, therefore, using a high permeability iron core is highly recommended to keep the input signals within applicable rates [21,26].

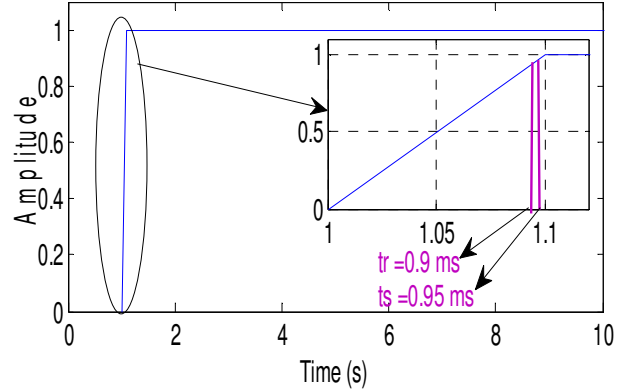

(a)

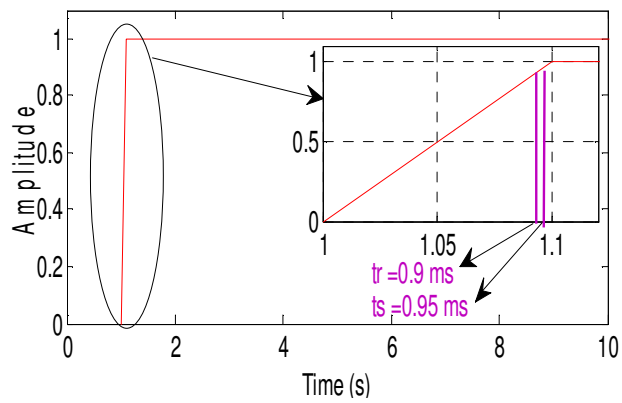

(b)

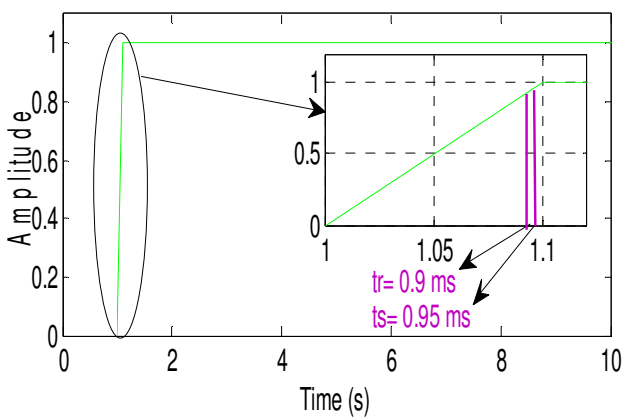

(c)

Fig. 7 Time response of system output

(a) Step response of $\Delta x(t)$

(b) Step response of $\Delta y(t)$

(c) Step response of $\Delta z(t)$

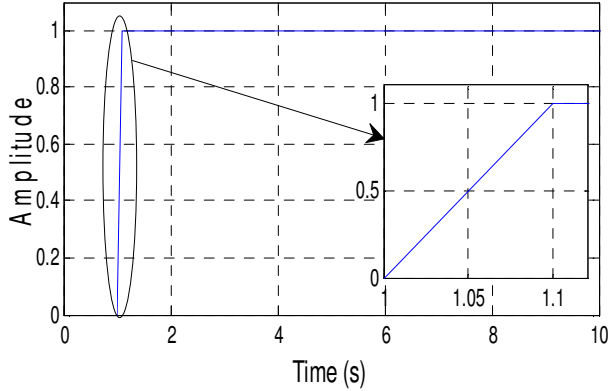

(a)

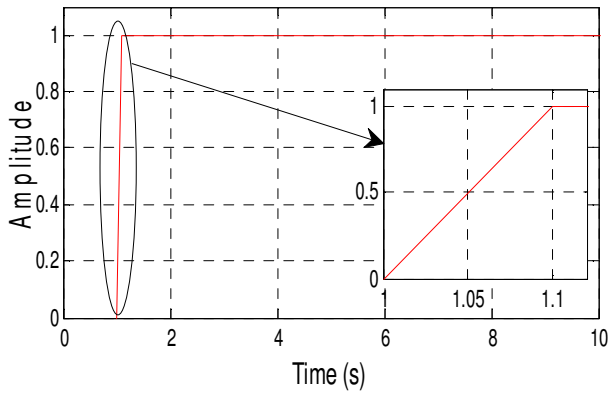

(b)

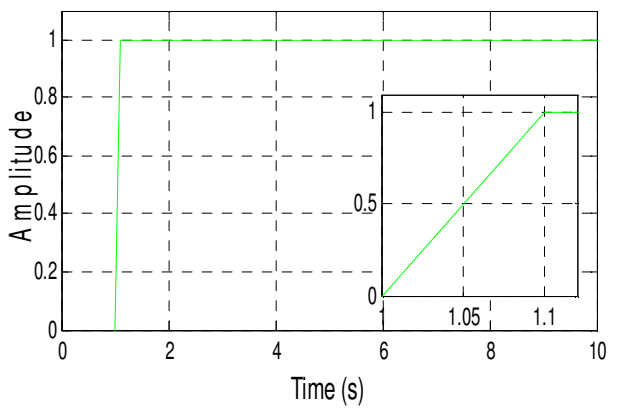

(c)

Fig. 8 Inputs of control system

(a) Control input $\Delta I_{1}(t)$

(b) Control input $\Delta I_{2}(t)$

(c) Control input $\Delta I_{3}(t)$ 
I.K. Mohammed, A.I. Abdulla: Design of optimised linear quadratic regulator for capsule endoscopes based on artificial bee colony tuning algorithm

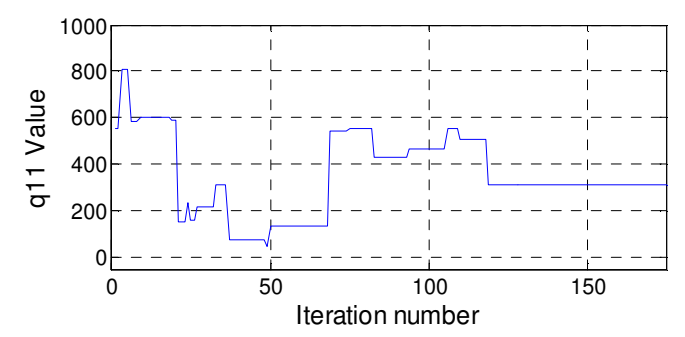

(a)

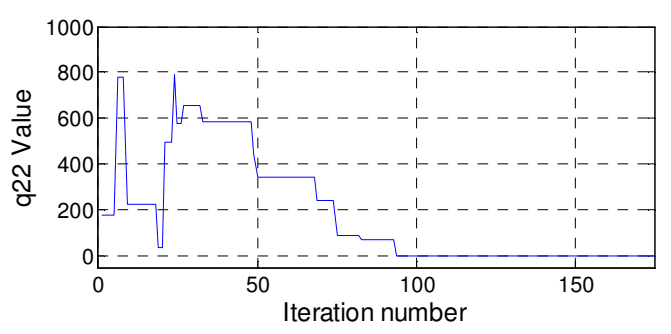

(b)

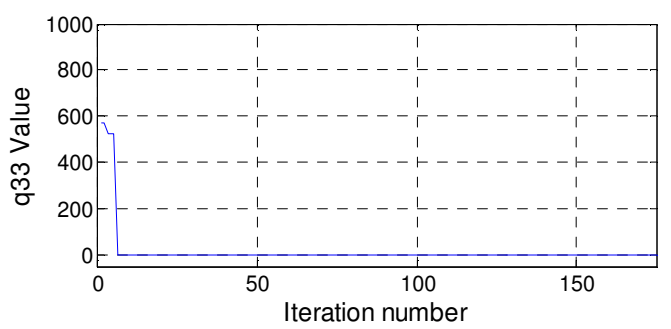

(c)

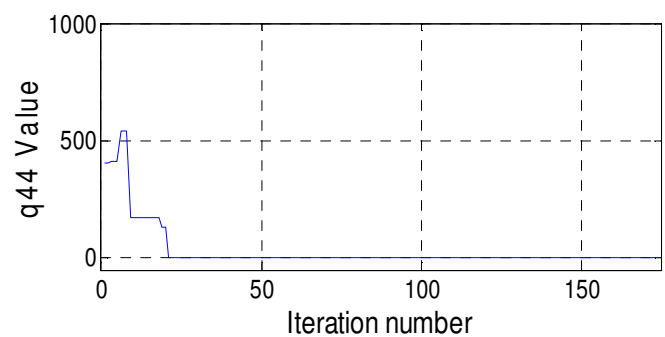

(d)

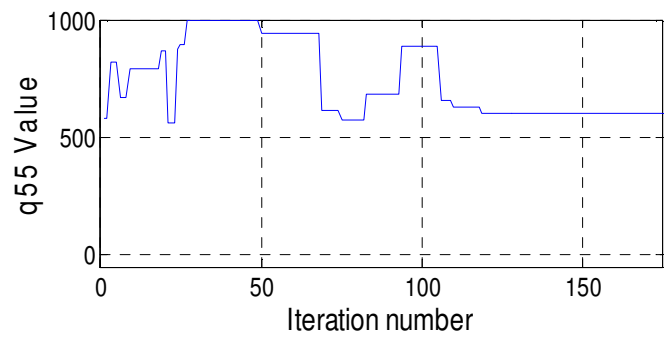

(e)

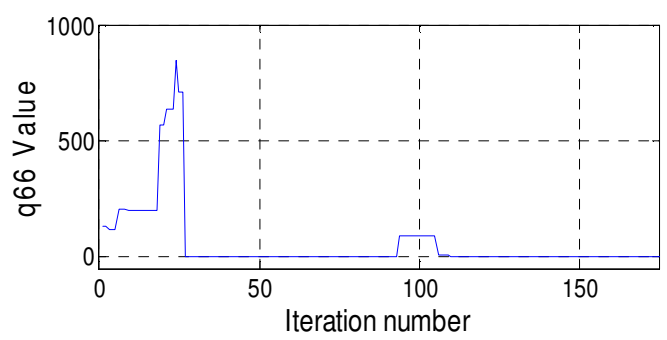

(f)

Fig. 9 Generation elements of $\boldsymbol{Q}$ matrix

(a) $q_{11}$ element; (b) $q_{22}$ element; (c) $q_{33}$ element;

(d) $q_{44}$ element; (e) $q_{55}$ element; (f) $q_{66}$ element

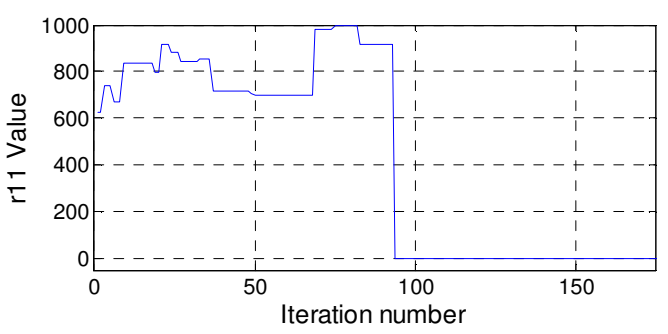

(a)

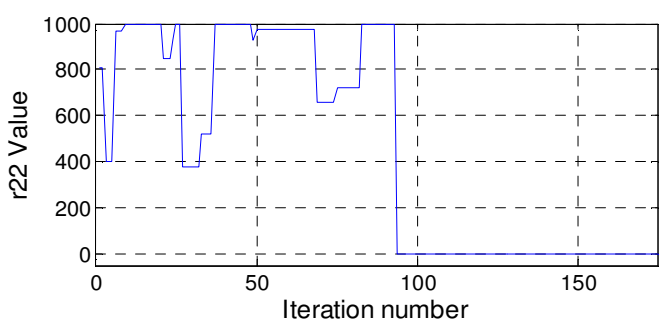

(b)

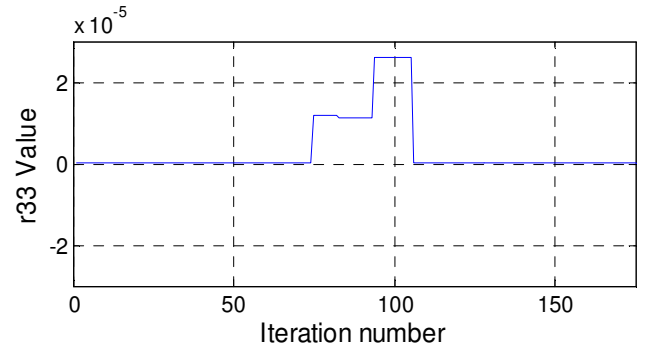

(c)

Fig. 10 Generation elements of $\boldsymbol{R}$ matrix

(a) $r_{11}$ element; (b) $r_{22}$ element; (c) $r_{33}$ element 


\section{CONCLUSIONS AND PROSPECTIVE RESEARCH}

This research contributes to the investigation into the development of controlled actuation methods for capsule endoscopes. The study focuses on the design of a 3DOF navigation system capable to guide the capsule through the digestive organs after suitable preparation. The dynamics of the control system is modelled mathematically and then formulated in state space form. Linear LQR controller was designed and simulated by using Matlab/Simulink environment to validate the proposed capsule actuation system. In this paper, the controller is optimised by obtaining the optimum controller gain parameters through the use of the ABC tuning algorithm. The performance of the optimised controller system was evaluated based on rise time, settling time, maximum overshoot, and control effort parameters. The simulation results showed that, based on acceptable control inputs, the auto tuning LQR-ABC controller under the unit step input was able to effectively provide a fast response with minimal overshoot value and steady state error. Consequently, the proposed LQR controller based on the $\mathrm{ABC}$ tuning algorithm controller can be adopted to design capsule navigation system. In prospective research, the validation of the proposed auto tuning LQR-ABC controller system by implementing it in real-time and by using a float point digital signal processor (DSP) will be considered.

\section{REFERENCES}

[1] W. Zhang, Y. Chen and P. Huang, Study on the system of a capsule endoscope driven by an outer rotational magnetic field, Proc. IEEE/ASME Int. Conf. on Mechatronic and Embedded Systems and Applications, pp. 1-5, 2006. DOI: 10.1109/MESA.2006.296925

[2] D. Chen, C. Hue, L. Wang and MQ.-H. Meng, Active actuation system of wireless capsule endoscope based on magnetic, Proc. IEEE Int. Conf. on Robotics and Biomimetics, pp. 99103, 2007. DOI: $10.1109 /$ ROBIO.2007.4522142

[3] M. Simi, P. Valdastri and C. Quaglia et al., Design, fabrication, and testing of a capsule with hybrid locomotion for gastrointestinal tract exploration, IEEE/ASME Transaction on mechatronics, Vol. 15, No. 2, pp. 170-180, 2010. DOI: 10.1109/TMECH.2010.2041244

[4] M. Sitti and S. Yim, Design and analysis of magnetically actuated and compliant capsule endoscope endoscopic robot, Proc. IEEE int. Conf. on Robotics and Automation, pp. 9-13, 2011. DOI: $10.1109 /$ ICRA.2011.5979819

[5] S. Yim and M. Sitti, Design and rolling locomotion of a magnetically actuated soft capsule endoscope, IEEE Trans. on Robotics, Vol. 28, No. 1, pp. 183-194, 2012.

DOI: $\underline{10.1109 / T R O .2011 .2163861 ~}$

[6] G.S. Lien, C.W. Liu and J.A. Jiang et al., Magnetic control system targeted for capsule endoscopic operations in the stomach-design, fabrication, and in vitro and ex vivo evaluations, IEEE Trans. on Biomed. Eng., Vol. 59, No. 7, pp. 2068-2079, 2012.

DOI: $10.1109 /$ TBME.2012.2198061

[7] F. Carpi, N. Kastelein and C. Pappone, Magnetically controllable gastrointestinal steering of video capsules, IEEE Trans. on Biomed. Eng., Vol. 58, No. 7, pp. 231-234, 2011.

DOI: 10.1109/TBME.2010.2087332 Epub 2010 Oct 14. 
[8] B. Ye, Ch Deng and Z. Sun et al., Fabrication of a novel handheld actuator for use in wireless capsule endoscope, Proc. IEEE Int. Conf. on Elect. Packaging Technology, pp. 1240-1242, 2013. DOI: 10.1109/ICEPT.2013.6756682

[9] X. Ye, C. Hasson and W.J. Yoon et al., Design of a steering mechanism for a tethered capsule endoscope, Proc. of IEEE Int. Conf. on CIS and RAM, pp. 234-238, 2015.

DOI: $10.1109 /$ ICCIS.2015.7274579

[10] Ch Lee, H. Choi and G. Go et al., Active locomotive intestinal capsule endoscope (ALICE): A prospective feasibility study, IEEE/ASME Trans. on Mechatronics, Vol. 20, No. 3, pp. 2067-2074, 2015. DOI: 10.1109/TMECH.2014.2362117

[11] W.A. Wangtrheem, Study on RF based wireless capsule endoscope, Proc. of the IEEE Int. Conf. on Mechatronics and Automation, pp. 1663-1667, 2006.

DOI: $10.1109 /$ ICMA.2006.257446

[12] I. Robandi, K. Nishimori, R. Nishimura and N. Ishihara, Optimal feedback control design using genetic algorithm in multimachine power system, Int. J. Elec. Power \& En. Sys., Vol. 23, No. 4, pp. 263-271, 2001. DOI: 10.1016/S0142-0615(00)00062-4

[13] J. Kennedy and R.C. Eberhart, Particle swarm optimization, Proceedings of the IEEE International Conference on Neural Networks, Vol. 4, pp. 1942-1948, IEEE Service Center, Piscat away, 1995. DOI: 10.1109/ICNN.1995.488968

[14] D. Srinivasan and T.H. Seow, Evolutionary Computation, CEC '03, Canberra, Australia, Vol. 4, pp. 2292-2297, 2003.

[15] D. Karaboga and B. Basturk, A powerful and efficient algorithm for numerical function optimization: artificial bee colony (ABC) algorithm, Journal of Global Optim., Vol. 39, No. 3, pp. 459-471, 2007. DOI: $10.1007 / \mathrm{s} 10898-007-9149-\mathrm{x}$

[16] A. Yurtkuran and E. Emel, An enhanced artificial bee colony algorithm with solution acceptance rule and probabilistic multisearch, Journal of Computational Intelligence and neuroscience, No. 41, pp. 1-13, 2016. DOI: $10.1155 / 2016 / 8085953$

[17] A. Abdulla, I. Mohammed and A. Jasim, Roll control system design using auto tuning LQR technique, International Journal of Engineering and Innovative, Vol. 6, No. 12, pp. 112$120,2016$.

[18] B. Basturk and D. Karaboga, An artificial bee colony (ABC) algorithm for numeric function optimization, IEEE Swarm Intelligence Symposium 2006, Indianapolis, Indiana, USA, 2006.

[19] D. Karaboga and B. Basturk, On the performance of artificial bee colony (ABC) algorithm, Applied Soft Computing, Vol. 8, No. 1, pp. 687-697, 2008.

DOI: $10.1016 /$ i.asoc.2007.05.007

[20] S. Tumanski, Induction coil sensors a review, MA: Oxford; 2007.

[21] I. Mohammed, B. Sharif and J. Neasham, Design and implementation of a magnetic levitation control system for robotically actuated capsule endoscopes, Proc. of the IEEE ROSE2012 conf., pp. 140-145, 2012. DOI: 10.1109/ROSE.2012.6402614

[22] M. Kaleemullah, W. Faris and F. Hasbullah, Design of robust $H \infty$, fuzzy and LQR controller for active suspension of a quarter car model, Proc. of the Int. Conf. on Mechatronics (ICOM), pp. 1-6, 2011. DOI 10.1109/ICOM.2011.5937197 
[23] A. Tewari, Modern Control Design with Matlab and Simulink, John Willey and Sons, LTD; 2005.

[24] F. Hasbullah and W. Faris, A Comparative analysis of LQR and fuzzy logic controller for active suspension using half car model, Proc. of the IEEE int. Conf. on Control Automation Robotics and Vision, pp. 2415-2420, 2010. DOI: 10.1109/ICARCV.2010.5707260

[25] D. Karaboga and B. Basturk, Artificial Bee Colony (ABC) optimization algorithm for solving constrained optimization problems, IFSA 2007, LNAI 4529, pp. 789-798, 2007. DOI: $10.1007 / 978-3-540-72950-177$

[26] I. Mohammed, B. Sharif and J. Neasham et al., Novel MIMO 4-DOF position control for capsule endoscope, Proc. of the IEEE Int. Symposium on Circuits and Systems (ISCAS), pp. 909-912, 2011. DOI: $10.1109 /$ ISCAS.2011.5937714

\section{A. APPENDIX A. DEFINITION OF THE MAGNET MOVEMENT EXPRESSION IN THE X- DIRECTION}

$$
\begin{aligned}
& x_{1}=(\sqrt{3} L / 4+x), x_{2}=(\sqrt{3} L / 4-x), y_{1}=(L / 4+y), y_{2}=(L / 4+y), \\
& D_{1}=\sqrt{3} x_{2}-y_{1}-2 \sqrt{3 z}, D_{2}=\sqrt{3} x_{1}+y_{1}+2 \sqrt{3} z, D_{3}=y_{2}-2 \sqrt{3} .
\end{aligned}
$$

\section{B. APPENDIX B. PARAMETERS EXPRESSION OF THE LINEARISED CAPSULE ACTUATION SYSTEM}

\section{B.1 IN THE X-DIRECTION}

$$
\begin{aligned}
& x_{01}=\left(-\sqrt{3} L / 4+x_{0}\right), x_{02}=\left(\sqrt{3} L / 4+x_{0}\right), y_{01}=\left(-L / 4+y_{0}\right), y_{02}=\left(L / 4+y_{0}\right), z_{0}=L / 2 \text {, } \\
& G_{1}=(K / M) I_{01}, G_{2}=(K / M) I_{02}, G_{3}=(K / M) I_{03}, R_{01}=\sqrt{x_{02}^{2}+y_{01}^{2}+z_{0}^{2}} \text {, } \\
& R_{02}=\sqrt{{x_{01}}^{2}+y_{01}{ }^{2}+z_{0}{ }^{2}}, R_{03}=\sqrt{{x_{0}}^{2}+y_{02}{ }^{2}+z_{0}{ }^{2}}, D_{01}=\sqrt{3} x_{02}-y_{01}-2 \sqrt{3} z_{0} \text {, } \\
& D_{02}=\sqrt{3} x_{01}+y_{01}+2 \sqrt{3} z_{0}, D_{03}=y_{02}-\sqrt{3} z_{0}, E_{0}=3 \sqrt{3} x_{02}+y_{01}+2 \sqrt{3} z_{0} \text {, } \\
& E_{01}=3 \sqrt{3} x_{01}+y_{01}+2 \sqrt{3} z_{0}, E_{02}=-\sqrt{3} x_{02}+3 y_{01}+2 \sqrt{3} z_{0}, E_{03}=\sqrt{3} x_{01}+3 y_{01}+2 \sqrt{3} z_{0} \text {, } \\
& E_{04}=3 y_{02}-\sqrt{3} z_{0}, E_{05}=2 \sqrt{3} y_{01}+z_{0}, E_{06}=\sqrt{3} y_{02}-z_{0}, E_{07}=-\sqrt{3} x_{02}+2 y_{01}+2 \sqrt{3} z_{0} \text {, } \\
& E_{08}=\sqrt{3} x_{01}+2 y_{01}+2 \sqrt{3} z_{0} \text {. } \\
& C_{11}=\frac{G_{1}}{I_{01}}\left[\frac{\left(\sqrt{3} y_{01}-x_{02}\right)}{R_{01}{ }^{5}}-\frac{5 x_{02} y_{01} D_{01}}{R_{01}{ }^{7}}\right], \quad C_{12}=\frac{G_{2}}{I_{02}}\left[\frac{\left(\sqrt{3} y_{01}-x_{01}\right)}{R_{02}{ }^{5}}-\frac{5 x_{01} y_{01} D_{02}}{R_{02}{ }^{7}}\right] \text {, } \\
& C_{13}=2 \frac{G_{3}}{I_{03}}\left[\frac{x_{0}}{R_{03}{ }^{5}}-\frac{5 x_{0} y_{02} D_{03}}{R_{03}{ }^{7}}\right] \text {, }
\end{aligned}
$$




$$
\begin{aligned}
& C_{14}=G_{1}\left[\frac{\left(R_{01}{ }^{2}+5 y_{01} E_{0}+5 x_{02}{ }^{2}\right)}{R_{01}{ }^{7}}+\frac{35 x_{02}{ }^{2} y_{01} D_{01}}{R_{01}{ }^{9}}\right]+ \\
& +G_{2}\left[\frac{\left(-R_{02}{ }^{2}+5 y_{01} E_{01}+5 x_{01}{ }^{2}\right)}{R_{02}{ }^{7}}-\frac{35 x_{01}{ }^{2} y_{01} D_{02}}{R_{02}{ }^{9}}\right]+ \\
& +2 G_{3}\left[\frac{\left(R_{03}{ }^{2}-5 y_{02} D_{03}-x_{0}{ }^{2}\right)}{R_{03}{ }^{7}}+\frac{35 x_{0}{ }^{2} y_{02} D_{03}}{R_{03}{ }^{9}}\right] \\
& C_{15}=G_{1}\left[\frac{\left(\sqrt{3} R_{01}{ }^{2}+5 x_{02} E_{02}-5 \sqrt{3} y_{01}{ }^{2}\right)}{R_{01}{ }^{7}}+\frac{35 x_{02} y_{01}{ }^{2} D_{01}}{R_{02}{ }^{7}}\right]+ \\
& +G_{2}\left[\frac{\left(-\sqrt{3} R_{02}{ }^{2}+5 x_{01} E_{03}+5 \sqrt{3} y_{01}{ }^{2}\right)}{R_{02}{ }^{7}}-\frac{35 x_{01} y_{01}{ }^{2} D_{02}}{R_{02}{ }^{9}}\right]+ \\
& +2 G_{3}\left[\frac{-5 x_{0} E_{04}}{R_{03}{ }^{7}}+\frac{35 x_{0} y_{02}{ }^{2} D_{03}}{R_{03}{ }^{9}}\right] \\
& C_{16}=G_{1}\left[\frac{\left(-5 \sqrt{3} y_{01} z_{0}+5 x_{02} E_{05}\right)}{R_{01}{ }^{7}} \frac{35 x_{02} y_{01} z_{0} D_{01}}{R_{01}{ }^{9}}\right]+ \\
& +G_{2}\left[\frac{\left(5 \sqrt{3} y_{01} z_{0}+5 x_{01} E_{05}\right)}{R_{02}{ }^{7}}-\frac{35 x_{01} y_{01} z_{0} D_{02}}{R_{02}{ }^{9}}\right]+ \\
& +2 G_{3}\left[\frac{5 x_{0} E_{06}}{R_{03}{ }^{7}}+\frac{35 x_{0} y_{02} z_{0} D_{03}}{R_{02}{ }^{9}}\right]
\end{aligned}
$$

\section{B.2 IN THE Y-DIRECTION}

$$
\begin{aligned}
C_{21}= & \frac{G_{1}}{I_{01}}\left[\frac{\left(\sqrt{3} y_{01}-x_{02}\right)}{R_{01}{ }^{5}}-\frac{5 x_{02} y_{01} D_{01}}{R_{01}{ }^{7}}\right], C_{22}=\frac{G_{2}}{I_{02}}\left[\frac{\left(\sqrt{3} y_{01}-x_{01}\right)}{R_{02}{ }^{5}}-\frac{5 x_{01} y_{01} D_{02}}{R_{02}{ }^{7}}\right], \\
C_{23}= & 2 \frac{G_{3}}{I_{03}}\left[\frac{E_{04}}{R_{03}{ }^{5}}-\frac{5 y_{02}{ }^{2} D_{03}}{R_{03}{ }^{7}}\right], C_{24}=C_{15}, \\
C_{25}= & G_{1}\left[\frac{\left(3 R_{01}{ }^{2}+15 y_{01} E_{07}\right.}{R_{01}{ }^{7}}+\frac{35 y_{01}{ }^{3} D_{01}}{R_{01}{ }^{9}}\right]+G_{2}\left[\frac{\left(-3 R_{02}{ }^{2}+15 y_{01} E_{08}\right.}{R_{02}{ }^{7}}-\frac{35 y_{01}{ }^{3} D_{02}}{R_{02}{ }^{9}}\right]+ \\
& +2 G_{3}\left[\frac{\left(3 R_{03}{ }^{2}+15 y_{02}\left(\sqrt{3} z_{0}-2 y_{02}\right)\right.}{R_{03}{ }^{7}}+\frac{35 y_{02}{ }^{3} D_{03}}{R_{03}{ }^{9}}\right],
\end{aligned}
$$




$$
\begin{aligned}
C_{26}= & G_{1}\left[\frac{\left(-2 \sqrt{3} R_{01}{ }^{2}+5 z_{0} E_{02}++10 \sqrt{3} y_{01}{ }^{2}\right)}{R_{01}{ }^{7}}+\frac{35 y_{01}{ }^{2} z_{0} D_{01}}{R_{01}{ }^{9}}\right]+ \\
& +G_{2}\left[\frac{\left(-2 \sqrt{3} R_{02}{ }^{2}+5 z_{0} E_{03}+10 \sqrt{3} y_{01}{ }^{2}\right)}{R_{02}{ }^{7}}-\frac{35 y_{01}{ }^{2} z_{0} D_{02}}{R_{02}{ }^{9}}\right]+ \\
& +2 G_{3}\left[\frac{\left(-\sqrt{3} R_{03}{ }^{2}-5 z_{0} E_{06}+5 \sqrt{3} y_{02}{ }^{2}\right)}{R_{03}{ }^{7}}-\frac{35 x_{0} y_{01}{ }^{2} D_{03}}{R_{03}{ }^{9}}\right]
\end{aligned}
$$

\section{B.3 IN THE Z-DIRECTION}

$$
\begin{aligned}
& C_{31}=-\frac{G_{1}}{I_{01}}\left[\frac{E_{05}}{R_{01}{ }^{5}}-\frac{5 y_{01} z_{0} D_{01}}{R_{01}{ }^{7}}\right], \quad C_{32}=-\frac{G_{2}}{I_{02}}\left[\frac{E_{05}}{R_{02}{ }^{5}}-\frac{5 y_{01} z_{0} D_{02}}{R_{02}{ }^{7}}\right], \quad C_{33}=-2 \frac{G_{3}}{I_{03}}\left[\frac{E_{06}}{R_{03}{ }^{5}}+\frac{5 y_{01} z_{0} D_{03}}{R_{03}{ }^{7}}\right] \\
& C_{34}=G_{1}\left[\frac{\left(5 x_{02} E_{05}-5 \sqrt{3} y_{01} z_{0}\right)}{R_{01}{ }^{7}}+\frac{35 x_{02} y_{01} z_{0} D_{01}}{R_{01}{ }^{9}}\right]+ \\
& +G_{2}\left[\frac{\left(5 x_{01} E_{05}+5 \sqrt{3} y_{01} z_{0}\right)}{R_{02}{ }^{7}}-\frac{35 x_{01} y_{01} z_{0} D_{02}}{R_{02}{ }^{9}}\right]+ \\
& +2 G_{3}\left[-\frac{35 x_{0} y_{02} z_{0} D_{03}}{R_{03}{ }^{7}}+\frac{5 x_{0} E_{06}}{R_{03}{ }^{9}}\right] \\
& C_{35}=G_{1}\left[\frac{\left(-2 \sqrt{3} R_{01}{ }^{2}+5 z_{0} E_{02}+10 \sqrt{3} y_{01}{ }^{2}\right)}{R_{01}{ }^{7}}+\frac{35 y_{01}{ }^{2} z_{0} D_{01}}{R_{01}{ }^{9}}\right]+ \\
& +G_{2}\left[\frac{\left(-2 \sqrt{3} R_{02}{ }^{2}+5 z_{0} E_{03}+10 \sqrt{3} y_{01}{ }^{2}\right)}{R_{02}{ }^{7}}-\frac{35 y_{01}{ }^{2} z_{0} D_{02}}{R_{02}{ }^{9}}\right]+ \\
& +2 G_{3}\left[\frac{\left(-\sqrt{3} R_{02}{ }^{2}-5 \sqrt{3} z_{0} E_{06}+5 \sqrt{3} y_{02}{ }^{2}\right)}{R_{03}{ }^{7}}+\frac{35 x_{0} y_{02}{ }^{2} D_{03}}{R_{03}{ }^{9}}\right] \\
& C_{36}=G_{1}\left[\frac{\left(-R_{01}{ }^{2}+5 z_{0}\left(6 \sqrt{3} y_{01}+z_{0}\right)\right)}{R_{01}{ }^{7}}+\frac{35 y_{01} z_{0}{ }^{2} D_{01}}{R_{01}{ }^{9}}\right]+ \\
& +G_{2}\left[-\frac{\left(-R_{02}{ }^{2}+5 z_{0}\left(6 \sqrt{3} y_{01}+z_{0}\right)\right)}{R_{02}{ }^{7}}+\frac{35 y_{01} z_{0}{ }^{2} D_{02}}{R_{02}{ }^{9}}\right]+ \\
& +2 G_{3}\left[\frac{\left(R_{03}{ }^{2}+5 z_{0}\left(3 \sqrt{3} y_{02}-z_{0}\right)\right)}{R_{03}{ }^{7}}+\frac{35 y_{02} z_{0}{ }^{2} D_{03}}{R_{03}{ }^{9}}\right]
\end{aligned}
$$

